Arq. Bras. Med. Vet. Zootec., v.65, n.2, p.513-518, 2013

\title{
Substituição de minerais inorgânicos por orgânicos na alimentação de poedeiras semipesadas
}

\author{
[Substitution of inorganic minerals for organic minerals in the \\ diet of semi heavy layers] \\ J.P. Figueiredo Júnior ${ }^{1}$, F.G.P. Costa ${ }^{1}$, P.E.N. Givisiez ${ }^{1}$, M.R. Lima ${ }^{1}$, J.H.V. Silva ${ }^{1}$, \\ D.F. Figueiredo-Lima ${ }^{2}$, E.P. Saraiva ${ }^{1}$, M.H.M. Santana ${ }^{1}$ \\ ${ }^{1}$ Universidade Federal da Paraíba - Areia, PB \\ ${ }^{2}$ Universidade Federal Rural de Pernambuco - Unidade Acadêmica de Garanhuns
}

\begin{abstract}
RESUMO
Avaliou-se o efeito da suplementação dietética de fontes orgânicas de microminerais para galinhas poedeiras sobre o desempenho e a qualidade do ovo. Foram utilizadas 216 aves de linhagem comercial, distribuídas num delineamento inteiramente ao acaso, com quatro tratamentos e nove repetições, com seis aves por parcela. O experimento teve duração de 140 dias, divididos em cinco ciclos de 28 dias. Os tratamentos consistiram de uma dieta basal formulada para atender às exigências nutricionais das aves, suplementada com minerais na forma inorgânica ou porcentagens de minerais orgânicos: T1 - Basal + $100 \%$ minerais inorgânicos; T2 - Basal + 100\% minerais orgânicos; T3 - Basal + 66\% minerais orgânicos; T4 - Basal + 33\% minerais orgânicos. A produção de ovos, peso do ovo, massa de ovo, conversão alimentar por massa e dúzia de ovo, porcentagem de albúmen, gravidade específica, espessura de casca e margem bruta relativa apresentaram melhores resultados quando os minerais inorgânicos são substituídos em $66 \%$ pelos orgânicos.
\end{abstract}

Palavras-chave: microminerais, ovos, quelatos, suplemento mineral

\begin{abstract}
This study evaluated the effect of the supplementation with organic microminerals on performance and egg quality of semi-heavy layers. Dekalk Brown layers $(n=216)$ were distributed according to a completely randomized design into four treatments, nine repetitions and six birds per repetition. The experiment lasted 140 days and was comprised of five 28 day cycles. The basal diet was formulated to fulfill the nutritional requirements and was supplemented with organic or inorganic minerals, resulting in four treatments: $T 1$ - basal $+100 \%$ inorganic minerals; $T 2$ - basal + 100\% organic minerals; T3 basal $+66 \%$ organic minerals; $T 4$ - basal $+33 \%$ organic minerals. Egg production, egg weight, egg mass, feed conversion for dozen and mass, albumen percentage, specific gravity, shell thickness and relative gross margin have better results when the inorganic minerals are replaced with $66 \%$ organic.
\end{abstract}

Keywords: chelates, eggs, microminerals, mineral supplementation

\section{INTRODUÇÃO}

Para se obter uma boa nutrição, é necessário que o animal receba quantidades adequadas de nutrientes, incluindo-se os minerais, a fim de evitar o comprometimento da sua saúde e do seu desempenho produtivo. Os microminerais são considerados de grande importância na alimentação das aves, pois participam de uma série de processos bioquímicos, essenciais ao crescimento e desenvolvimento, destacando a formação óssea (Brito et al., 2006). Dessa forma, várias tentativas têm sido feitas para torná-los mais biodisponíveis, ao protegê-los das condições do trato gastrintestinal.

Recebido em 16 de agosto de 2011

Aceito em 1 de outubro de 2012

E-mail: peudure@hotmail.com 
Os minerais orgânicos ou minerais quelatados vêm sendo estudados por vários pesquisadores, pois podem apresentar melhor biodisponibilidade em relação aos minerais inorgânicos (Maciel et al., 2010). Eles proporcionam maior rapidez na absorção e seu transporte é facilitado, resultando em melhorias na vida útil das aves e na qualidade dos ovos. Partindo do pressuposto de que são mais facilmente absorvidos e retidos pelas aves, os minerais orgânicos podem ser adicionados a uma concentração muito mais baixa na dieta do que minerais inorgânicos, sem qualquer efeito negativo sobre o desempenho produtivo, e podem, potencialmente, reduzir a excreção de minerais (Nollet et al., 2007), diminuindo a poluição ambiental. Todavia, os resultados ainda são contraditórios e, portanto, novos estudos devem ser realizados com o intuito de avaliar a resposta das aves aos minerais quelatados em relação aos minerais inorgânicos.

Objetivou-se com este trabalho avaliar índices de desempenho e qualidade de ovos de poedeiras suplementadas com fontes orgânicas e inorgânicas de zinco, cobre, manganês, ferro e selênio, bem como realizar a análise econômica dos tratamentos.

\section{MATERIAL E MÉTODOS}

O experimento teve duração de 140 dias, divididos em cinco períodos de 28 dias cada. Foram utilizadas 216 aves da linhagem Dekalb Brown a partir de 68 semanas de idade, alojadas em galpão convencional de postura, com comedouros tipo calha e bebedouros tipo niple, sendo agrupadas em gaiolas de arame galvanizado com dimensões de $24 \mathrm{~cm}$ x $37 \mathrm{~cm} \mathrm{x}$ $41 \mathrm{~cm}$, onde receberam água e ração à vontade. $\mathrm{O}$ programa de luz adotado foi de 16 horas de luz (natural + artificial).

Os tratamentos consistiram em quatro dietas, com a substituição da fonte mineral inorgânica por níveis de fonte mineral orgânica (Tab. 1). As dietas foram formuladas de acordo com as recomendações de Rostagno et al. (2005). O delineamento utilizado foi o inteiramente ao acaso, distribuído em quatro tratamentos e nove repetições de seis aves por unidade experimental para cada período experimental.
Para avaliação da qualidade do albúmen, os ovos foram pesados individualmente em balança de precisão, sendo posteriormente quebrados sobre uma mesa especial de vidro e medida a altura do albúmen através de um altímetro especial AMES. A unidade Haugh foi calculada de acordo com Card e Nesheim (1968), através da equação $\mathrm{UH}=100 \log \left(\mathrm{H}+7,57-1,7 \mathrm{~W}^{0,37}\right)$, onde: $\mathrm{UH}=$ unidade Haugh; $\mathrm{H}=$ altura de albúmen $(\mathrm{mm}) ; \mathrm{W}=$ peso do ovo $(\mathrm{g})$.

Realizou-se análise econômica através da margem bruta relativa (MBR), em que se avalia a margem bruta (MB) dos tratamentos que utilizaram minerais orgânicos (T2, T3 e T4) em relação à $\mathrm{MB}$ do tratamento controle (T1). A determinação da MBR foi realizada considerando somente os custos variáveis de arraçoamento, uma vez que os custos fixos foram iguais para todos os tratamentos. Para esses cálculos foram considerados o consumo de ração (CR, kg/ave no período experimental), o número de dúzias de ovos produzidas (DP, dz/ave) e a massa de ovo produzida (MOP, kg/ave) pelas poedeiras durante os 140 dias de duração do experimento. Com os preços dos insumos, calcularam-se os custos por quilograma de cada ração experimental (CQR, US\$/kg), o custo de arraçoamento (CAR, US\$/ave), os custos de arraçoamento por dúzia de ovos (CAD, US\$/dz) e por quilograma de ovo (CAQ, US\$/kg), respectivamente. A renda bruta (RB, US\$) foi calculada por meio da multiplicação do número de dúzias produzidas no período pelo preço da dúzia de ovos (cotação Jox Assessoria Agropecuária = US\$23,18/caixa com 30 dúzias; Cotação Dólar Bacen $=\mathrm{R} \$ 1,71(22 / 11 / 2010))$. A margem bruta (MB, US\$) de cada tratamento foi calculada pela diferença entre renda bruta e o custo de arraçoamento. Finalmente, calculou-se a margem bruta relativa (MBR, \%), que correspondeu à margem bruta de cada tratamento em relação ao tratamento controle.

As análises estatísticas das características avaliadas foram realizadas utilizando-se o pacote computacional SAEG 8.0 (Manual..., 2004). As médias foram comparadas pelo teste Tukey a $5 \%$ de probabilidade. 
Substituição de minerais...

Tabela 1. Composição percentual e níveis nutricionais calculados das rações experimentais utilizadas para poedeiras

\begin{tabular}{|c|c|c|c|c|}
\hline \multirow{3}{*}{ Ingrediente } & \multicolumn{4}{|c|}{ Tratamentos } \\
\hline & $100 \%$ & $100 \%$ & $66 \%$ & $33 \%$ \\
\hline & Inorgânico & Orgânico & Orgânico & Orgânico \\
\hline Milho & 57,791 & 58,141 & 58,262 & 58,379 \\
\hline Farelo de soja & 26,134 & 26,067 & 26,043 & 26,020 \\
\hline Calcário & 9,700 & 9,701 & 9,701 & 9,702 \\
\hline Óleo de soja & 3,610 & 3,491 & 3,450 & 3,411 \\
\hline Fosfato bicálcico & 1,509 & 1,508 & 1,508 & 1,507 \\
\hline Min. Inorgânico ${ }^{1}$ & 0,400 & 0,000 & 0,000 & 0,000 \\
\hline Bioplex Fe & 0,000 & 0,027 & 0,0176 & 0,0088 \\
\hline Bioplex $\mathrm{Cu}$ & 0,000 & 0,010 & 0,0066 & 0,0033 \\
\hline Bioplex Mn & 0,000 & 0,080 & 0,0528 & 0,0264 \\
\hline Bioplex Zn & 0,000 & 0,037 & 0,0242 & 0,0121 \\
\hline Selplex & 0,000 & 0,013 & 0,0083 & 0,0042 \\
\hline Iodato de $\mathrm{Ca}$ & 0,000 & 0,0007 & 0,0007 & 0,0007 \\
\hline DL-Metionina & 0,237 & 0,237 & 0,237 & 0,237 \\
\hline L-Lisina & 0,007 & 0,008 & 0,009 & 0,008 \\
\hline Suplemento vitamínico ${ }^{2}$ & 0,050 & 0,050 & 0,050 & 0,050 \\
\hline Cloreto de colina $60 \%$ & 0,060 & 0,060 & 0,060 & 0,060 \\
\hline Sal comum & 0,491 & 0,491 & 0,491 & 0,491 \\
\hline Antioxidante $^{3}$ & 0,010 & 0,010 & 0,010 & 0,010 \\
\hline Total & 100,000 & 100,000 & 100,000 & 100,000 \\
\hline \multicolumn{5}{|l|}{ Composição química } \\
\hline Energia metabolizável (kcal/kg) & 2.900 & 2.900 & 2.900 & 2.900 \\
\hline Proteína bruta $(\%)$ & 17,00 & 17,00 & 17,00 & 17,00 \\
\hline Lisina digestível (\%) & 0,784 & 0,784 & 0,784 & 0,784 \\
\hline Metionina+cistina digestível (\%) & 0,713 & 0,713 & 0,713 & 0,713 \\
\hline Metionina digestível (\%) & 0,478 & 0,478 & 0,478 & 0,478 \\
\hline Cálcio (\%) & 4,2 & 4,2 & 4,2 & 4,2 \\
\hline Fósforo disponível (\%) & 0,375 & 0,375 & 0,375 & 0,375 \\
\hline Cloro $(\%)$ & 0,348 & 0,348 & 0,348 & 0,348 \\
\hline Sódio (\%) & 0,230 & 0,230 & 0,230 & 0,230 \\
\hline
\end{tabular}

${ }^{\mathrm{I}}$ Premix mineral inorgânico por kg de produto: Mn, 20g; Fe, 10g; Zn, 13,7g; Cu, 2,5g; Se, 0,063g; I, 0,19g; e veículo q.s.p., 500g. ${ }^{2}$ Premix vitamínico por kg de ração: vit. A - 15.000.000Ul, vit. $\mathrm{D}_{3}-1.500 .000 \mathrm{Ul}$, vit. $\mathrm{E}-15.000 \mathrm{Ul}$, vit. $\mathrm{B}_{1}-2,0 \mathrm{~g}$, vit. $\mathrm{B}_{2}-4,0 \mathrm{~g}$, vit. $\mathrm{B} 6-3,0 \mathrm{~g}$, vit. $\mathrm{B}_{12}-0,015 \mathrm{~g}$, Ácido nicotínico $-25 \mathrm{~g}$, Ácido pantotênico - $10 \mathrm{~g}$, vit. $\mathrm{K}_{3}$ $-3,0 \mathrm{~g}$, Ácido fólico - 1,0 g, Bacitracina de zinco - 10g, Selênio - 250mg, Antioxidante BHT - 10g, e veículo. q.s.p. $-1.000 \mathrm{~g} .{ }^{3}$ Etoxiquim $-10 \mathrm{~g}$, e veículo q.s.p. $-1.000 \mathrm{~g}$.

\section{RESULTADOS E DISCUSSÃO}

Nas Tab. 2 e 3 são apresentadas as médias das variáveis produtivas e de qualidade dos ovos de poedeiras semipesadas a partir de 68 semanas de idade. Foi verificado efeito significativo $(\mathrm{P}<0,05)$ entre os tratamentos estudados sobre as variáveis produtivas, exceto para consumo de ração $(\mathrm{P}>0,05)$. Com relação às variáveis qualitativas, a PA, EC e GE foram influenciadas significativamente $\quad(\mathrm{P}<0,05), \quad$ não $\quad$ sendo encontrado qualquer efeito nas demais variáveis estudadas, apresentando todas as características comportamento condizente aos padrões da
Linhagem (Manual..., 2009) para a fase em estudo.

A ausência de diferença significativa $(P>0,05)$ para a variável $C R$ indica que a forma de suplementação mineral na dieta não influenciou os tratamentos. A suplementação com porcentagens acima da recomendação de minerais, como zinco, cobre e selênio, pode ocasionar diminuição no consumo de ração. A redução no consumo de ração diário e percentual de postura podem servir como parâmetro de sinais de intoxicação de selênio para poedeiras (Payne et al., 2005). 
Tabela 2. Médias de consumo de ração (CR), produção de ovos (PR), peso médio dos ovos (PO), massa de ovo (MO), conversão alimentar por massa (CAMO) e por dúzia de ovos (CADZ), segundo os níveis e as fontes de suplementação dos microminerais

\begin{tabular}{lcccccc} 
Tratamentos & $\begin{array}{c}\text { CR } \\
\text { (g/ave/dia) }\end{array}$ & $\begin{array}{c}\text { PR } \\
(\%)\end{array}$ & $\begin{array}{c}\text { PO } \\
(\mathrm{g})\end{array}$ & $\begin{array}{c}\text { MO } \\
(\mathrm{g} / \mathrm{ave} / \mathrm{dia})\end{array}$ & $\begin{array}{c}\text { CAMO } \\
(\mathrm{g} / \mathrm{g})\end{array}$ & $\begin{array}{c}\text { CADZ } \\
(\mathrm{kg} / \mathrm{dz})\end{array}$ \\
\hline 100\% Inorgânico & 101,79 & $79,54 \mathrm{c}$ & $65,82 \mathrm{~b}$ & $52,36 \mathrm{~b}$ & $1,94 \mathrm{a}$ & $1,54 \mathrm{a}$ \\
$100 \%$ Orgânico & 102,51 & $82,04 \mathrm{~b}$ & $67,10 \mathrm{ab}$ & $55,05 \mathrm{a}$ & $1,86 \mathrm{~b}$ & $1,50 \mathrm{ab}$ \\
66\% Orgânico & 101,72 & $83,37 \mathrm{a}$ & $67,21 \mathrm{ab}$ & $56,03 \mathrm{a}$ & $1,82 \mathrm{~b}$ & $1,46 \mathrm{~b}$ \\
$33 \%$ Orgânico & 103,02 & $83,25 \mathrm{ab}$ & $67,74 \mathrm{a}$ & $56,39 \mathrm{a}$ & $1,83 \mathrm{~b}$ & $1,49 \mathrm{~b}$ \\
\hline C.V. (\%) & 1,56 & 1,23 & 2,09 & 2,40 & 2,81 & 1,97 \\
\hline
\end{tabular}

Valores seguidos por letras distintas nas colunas indicam diferenças pelo Teste Tukey $(\mathrm{P}<0,05) ; \mathrm{C} . \mathrm{V} .=$ coeficiente de variação.

Tabela 3. Médias de porcentagem de gema (PG), albúmen (PA) e casca (PC), gravidade específica (GE), espessura de casca (EC) e qualidade do albúmen (UH), segundo os níveis e as fontes de suplementação dos microminerais

\begin{tabular}{lcccccc}
\hline \multicolumn{1}{c}{ Tratamentos } & PG & PA & PC & GE & EC & UH \\
& $(\%)$ & $(\%)$ & $(\%)$ & $(\mathrm{g} / \mathrm{mL})$ & $(\mathrm{mm})$ & $\begin{array}{c}\text { (Haugh) } \\
\text { 100\% Inorgânico }\end{array}$ \\
\hline 100\% Orgânico & 25,98 & $62,61 \mathrm{a}$ & 8,95 & $1,0779 \mathrm{~b}$ & $0,447 \mathrm{~b}$ & 84,12 \\
66\% Orgânico & 26,30 & $62,08 \mathrm{ab}$ & 8,98 & $1,0801 \mathrm{a}$ & $0,471 \mathrm{a}$ & 85,56 \\
33\% Orgânico & 26,65 & $61,68 \mathrm{~b}$ & 9,06 & $1,0803 \mathrm{a}$ & $0,483 \mathrm{a}$ & 84,05 \\
\hline C.V. $(\%)$ & 2,58 & 1,04 & 3,46 & 0,14 & 2,96 & 3,07 \\
\hline
\end{tabular}

Valores seguidos por letras distintas nas colunas indicam diferenças pelo Teste Tukey $(\mathrm{P}<0,05)$; C.V. = coeficiente de variação.

O tratamento com minerais $100 \%$ inorgânico apresentou maior $(\mathrm{P}<0,05)$ percentagem de albúmen em relação ao tratamento com $33 \%$ orgânico, não havendo diferença estatística entre os demais tratamentos. Esses resultados diferem dos de Saldanha et al. (2009), que utilizaram diferentes fontes e porcentagens de elementos traços - Fe, $\mathrm{Zn}, \mathrm{Cu}, \mathrm{Mn}$, I e Se - na dieta e não encontraram influência significativa na porcentagem de albúmen entre tratamentos de poedeiras semipesadas. Esses resultados podem ser explicados porque as porcentagens de microminerais aplicadas atualmente nas dietas do mercado brasileiro são mais do que as exigidas pelas aves, não afetando, portanto, a variável qualitativa.

Não houve efeito significativo ( $\mathrm{P}>0,05)$ entre os tratamentos para PG, PC e UH. Saldanha et al. (2009) afirmam que a qualidade interna dos ovos, representada pelos parâmetros de porcentagem de gema e unidade Haugh, não foi significativamente influenciada pelos tratamentos com suplementação de minerais orgânicos ou inorgânicos. Do mesmo modo, Swiatkiewicz e Koreleski (2008) suplementaram as dietas com microminerais orgânicos isolados e/ou associados e não observaram qualquer efeito sobre a variável PC.

Fernandes et al. (2008) observaram tendência de melhor peso de gema, indicando efeito positivo dos minerais na absorção e/ou proteção das vitaminas lipossolúveis quando galinhas HyLine com 67 semanas de idade foram alimentadas com zinco, manganês e selênio orgânico.

Segundo Maciel et al. (2010), a porcentagem de casca não é alterada pela utilização de fontes orgânicas na dieta. Porém, Lundeen (2001) observou melhora na porcentagem da casca no período de 40 - 60 semanas de idade das aves ao utilizar proteinato de $\mathrm{Zn}$ e Mn. Hudson et al. (2004) observaram melhor qualidade de casca quando matrizes com 58 semanas de idade foram suplementadas com porcentagens de zinco acima do recomendado.

De acordo com Brito et al. (2006), as possíveis causas para superioridade dos índices produtivos nos tratamentos que receberam suplementação com minerais orgânicos seriam a utilização em menor quantidade dos ingredientes minerais para atender às necessidades nutricionais das aves e ao melhor aproveitamento destes - maior taxa de 
absorção e/ou menor quantidade de interações entre os minerais - ou mesmo esses dois fatores associados.

$\mathrm{Na}$ formulação de premixes vitamínicos e minerais são utilizadas porcentagens de microminerais acima do exigido a fim de garantir ampla margem de segurança nas dietas, tornando impossível detectar quaisquer benefícios adicionais, independentemente da forma de apresentação do mineral, quer orgânico ou inorgânico (Fernandes et al., 2008).

A substituição de minerais inorgânicos por orgânicos proporcionou maiores resultados de renda bruta e margem bruta, além de menor custo da dúzia de ovo produzido, aumentando com isso a rentabilidade econômica do produtor de aves de postura (Tab. 4).

A margem bruta relativa apresentou valores aproximados para os tratamentos T3 (66\% org.) e T4 (33\% org.). O tratamento que utilizou $66 \%$ de microminerais na forma orgânica apresentou melhores resultados quando comparado aos demais, demonstrando menor custo de arraçoamento (CAR, CAQ (US\$/kg) e CAD (US\$/dz)) e maior número de dúzias produzidas (DP, dz/ave), resultando em maior rentabilidade para atividade desenvolvida.

Tabela 4. Análise econômica da substituição de minerais inorgânicos por orgânicos na alimentação de poedeiras

\begin{tabular}{ccccc} 
& T1 & T2 & T3 & T4 \\
& (100\% Inorg.) & $(100 \%$ Org.) & (66\% Org.) & (33\% Org.) \\
\hline CR (kg/ave) & 14,25 & 14,35 & 14,24 & 14,42 \\
CQR (US\$/kg) & 0,38 & 0,38 & 0,37 & 0,37 \\
CAR (US\$/kg) & 5,41 & 5,45 & 5,27 & 5,33 \\
DP (dz/ave) & 9,28 & 9,57 & 9,73 & 9,71 \\
CAD (US\$/dz) & 0,58 & 0,57 & 0,54 & 0,55 \\
MOP (kg/ave) & 7,33 & 7,71 & 7,84 & 7,89 \\
CAQ (US\$/kg) & 0,74 & 0,71 & 0,67 & 0,68 \\
RB (US\$) & 7,15 & 7.37 & 7,49 & 7,48 \\
MB (US\$) & 1,74 & 1,92 & 2,22 & 2,15 \\
\hline MBR (\%) & 100 & 110,34 & 127,59 & 123,56 \\
\hline
\end{tabular}

$\mathrm{CR}=$ consumo de ração; $\mathrm{CQR}=$ custo por quilograma de ração; $\mathrm{CAR}=$ custo de arraçoamento; $\mathrm{DP}=$ dúzias de ovos produzidas; $\mathrm{CAD}=$ custo de arraçoamento por dúzia de ovos; $\mathrm{MOP}=$ massa de ovo produzida; $\mathrm{CAQ}=$ custo de arraçoamento por quilograma de ovo; $\mathrm{RB}=$ renda bruta; $\mathrm{MB}=$ margem bruta; $\mathrm{MBR}=$ margem bruta relativa.

Os tratamentos com $100 \%$ e $33 \%$ de microminerais na forma orgânica mostraram menor rentabilidade do que o tratamento com $66 \%$. O custo de arraçoamento total foi maior para o tratamento alimentado com $100 \%$ de microminerais orgânicos devido ao maior consumo de ração, apesar de o preço por quilograma da ração ter sido semelhante ao dos demais tratamentos. A rentabilidade foi também menor em virtude da menor produção de ovos. É pouco provável que o pior desempenho tenha sido por causa da interação de microminerais orgânicos e indisponibilidade de alguns destes. Pode ter ocorrido elevada absorção dos minerais, resultando em intoxicação e comprometimento da produção. Em relação ao tratamento com $33 \%$ de minerais orgânicos, provavelmente não foram atendidas as necessidades dos animais, sendo necessário maior consumo de ração para suprimento das necessidades nutricionais e, consequentemente, onerando os custos do tratamento.

Esses dados confirmam a eficiência da forma orgânica em melhorar a disponibilidade dos minerais, fazendo com que as aves tenham melhor aproveitamento das rações e consequente melhora nos índices produtivos, permitindo ao produtor atingir os seus objetivos de maximização de lucros e minimização de custos.

\section{CONCLUSÕES}

A adição de $66 \%$ de minerais orgânicos em substituição aos inorgânicos promove melhores resultados produtivos, como também melhor margem bruta. 


\section{REFERÊNCIAS}

BRITO, J.A.G.; BERTECHINI, A.G.; FASSANI, E.J. et al. Uso de microminerais sob a forma de complexo orgânico em rações de frangas de reposição no período de 7 a 12 semanas de idade. Rev. Bras. Zootec., v.35, p.1342-1348, 2006.

CARD, L.E.; NESHEIM, M.C. Produccion avicola. New York: Ithaca, 1968. 394p.

FERNANDES, J.I.M.; MURAKAMI, A.E.; SAKAMOTO, M.I. et al. Effects of organic mineral dietary supplementation on production performance and egg quality of white layers. Rev. Bras. Cienc. Avic., v.10, p.59-65, 2008.

HAMILTON, R.M.G. Methods and factors that affect the measurement of egg shell quality. Poult. Sci., v.61, p.2002-2039, 1982.

HUDSON, B.P.; DOZIER, W.A.; WILSON, J.L. et al. Reproductive performance and immune status of caged broiler breeder hens provided diets supplemented with either inorganic or organic sources of zinc from hatching to $65 \mathrm{wk}$ of age. J. Appl. Poult. Res., v.13, p.349-359, 2004.

LEESON, S.; SUMMERS, J.D. Nutrition of the chicken. Ontario: University Books, 2001. 591p.

LIM, H.S.; PAIK, I.K. Effects of supplementary mineral methionine chelates $(\mathrm{Zn}, \mathrm{Cu}, \mathrm{Mn})$ on the performance and eggshell quality of laying hens. Asian Austral. J. Anim., v.16, p.1804-1808, 2003.

LUNDEEN, T. Mineral proteinates may have positive effect on shell quality. Feedstuffs, v.73, p.10-15, 2001.

MACIEL, M.P.; SARAIVA, E.P.; AGUIAR, E.F. et al. Effect of using organic microminerals on performance and external quality of eggs of commercial laying hens at the end of laying. Rev. Bras. Zootec., v.39, p.344-348, 2010.

MANUAL da linhagem dekalb brown. Granja Planalto. Uberlândia: Minas Gerais, 2009, 42p.

MANUAL de utilização do programa SAEG Sistema de análise estatística e genética. Versão 8.0. Viçosa: Universidade Federal de Viçosa, 2004, 59p.
NOLLET, L.; VAN DER KLIS, J.D.; LENSING, M. et al. The effect of replacing inorganic with organic trace minerals in broiler diets on productive performance and mineral excretion. J. Appl. Poult. Res., v.16, p.592-597, 2007.

PAIK, I. Application of chelated minerals in animal production. Asian Austral. J. Anim., v.14, p.191-198, 2001.

PAIK, I.; LEE, H.; PARK, S. Effects of organic iron supplementation on the performance and iron content in the egg yolk of laying hens. J. Poult. Sci., v.46, p.198-202, 2009.

PAYNE, R.L.; LAVERGNE, T.K.; SOUTHERN, L.L. Effect of inorganic versus organic selenium on hen production and egg selenium concentration. Poult. Sci., v.84, p.232-237, 2005.

ROSTAGNO, H.S.; ALBINO, L.F.T.; DONZELE, J.L. et al. Tabelas Brasileiras para Aves e Suínos: Composição de alimentos e exigências nutricionais. Viçosa: Universidade Federal de Viçosa, 2005. 186p.

SALDANHA, E.S.P.B.; GARCIA, E.A.; PIZZOLANTE, C.C. et al. Effect of organic mineral supplementation on the egg quality of semiheavy layers in their second cycle of lay. Rev. Bras. Cienc. Avic., v.11, p.215-222, 2009.

SCOTT, M.L.; NESHEIM, M.C.; YOUNG, R.J. Nutrition of the chicken. New York: M.L. Scott, 1982. 562p.

SECHINATO, A.S.; ALBUQUERQUE, R.; NAKADA, S. Efeito da suplementação dietética com micro minerais orgânicos na produção de galinhas poedeiras. Braz. J. Vet. Res. Anim. Sci., v.43, p.159166, 2006.

SWIATKIEWICZ, S.; KORELESKI, J. The effect of zinc and manganese source in the diet for laying hens on eggshell and bones quality. Vet. Med-Checa, v.53, p.553-563, 2008.

TRINDADE NETO, M.A.; PACHECO, B.H.C.; ALBUQUERQUE, R. et al. Lysine and zinc chelate in diets for brown laying hens: effects on egg production and composition. Rev. Bras. Zootec., v.40, p.377-384, 2011. 